\title{
Surgical removal of an exceedingly rare papillary fibroelastoma of the aortic wall causing unstable angina
}

Paolo Masiello ${ }^{1}$, Angelo Catalano ${ }^{2}$, Generoso Mastrogiovanni ${ }^{1}$, Geppina Eusebio ${ }^{2}$, Anna

Maria De Roberto ${ }^{2}$, Pierpaolo Chivasso ${ }^{1}$, Giuseppe Ciancia ${ }^{1}$, Ivana Iesu ${ }^{1}$, Donato

Triggiani $^{1}$, and Severino Iesu ${ }^{1}$

${ }^{1}$ University Hospital 'San Giovanni di Dio e Ruggi d'Aragona'

${ }^{2}$ Maria Santissima Addolorata Hospital

March 26, 2021

\begin{abstract}
Papillary fibroelastomes are rare benign neoformations usually originating from the valvular endocardium, presenting with cerebral ischemia and/or myocardial infarction due to embolization from the mass. We report an exceedingly rare case of aortic wall papillary fibroelastoma simulating unstable angina, diagnosed with trans-thoracic/trans-esophageal echocardiography and CT scan and surgically successfully removed
\end{abstract}

Surgical removal of an exceedingly rare papillary fibroelastoma of the aortic wall causing unstable angina

Running title: Papillary fibroelastoma of the aortic wall

Paolo Masiello ${ }^{1^{*}} \mathrm{MD}$, Angelo Catalano ${ }^{2} \mathrm{MD}$, Generoso Mastrogiovanni ${ }^{1} \mathrm{MD}$, Geppina Eusebio ${ }^{2} \mathrm{MD}$, Anna Maria De Roberto ${ }^{2} \mathrm{MD}$, Pierpaolo Chivasso ${ }^{1} \mathrm{MD}$, Giuseppe Ciancia ${ }^{3} \mathrm{MD}$, Ivana $\mathrm{Iesu}^{4} \mathrm{MD}$, Donato Triggiani ${ }^{1} \mathrm{MD} \mathrm{PhD}$, Severino Iesu ${ }^{1} \mathrm{MD}$

${ }^{1}$ Emergency Cardiac Surgery, Cardio-Thoracic and Vascular Department, University Hospital "San Giovanni di Dio e Ruggi D'Aragona" - Salerno - Italy

${ }^{2}$ Cardiology Division, Emergency Department Eboli-Battipaglia-Roccadaspide, Maria SS Addolorata General Hospital -Eboli - Italy

${ }^{3}$ Pathological Anatomy and Histology, Onco-Hematological Department, University Hospital "San Giovanni di Dio e Ruggi D'Aragona" - Salerno - Italy

${ }^{4}$ Clinical Cardiology Division, Cardio-Thoracic-Vascular Department, "San Giovanni di Dio e Ruggi D'Aragona" University Hospital of Salerno - Italy

*Corresponding Author:

Paolo Masiellohttps://orcid.org/0000-0002-5226-3078

Piazza Vittorio Veneto 39

84123 - Salerno - Italy

Mobile +39 3389499910

paolo.masiello1@virgilio.it 


\title{
Sources of funding: none
}

\begin{abstract}
:
Papillary fibroelastomes are rare benign neoformations usually originating from the valvular endocardium, presenting with cerebral ischemia and/or myocardial infarction due to embolization from the mass. We report an exceedingly rare case of aortic wall papillary fibroelastoma simulating unstable angina, diagnosed with trans-thoracic/trans-esophageal echocardiography and CT scan and surgically successfully removed.
\end{abstract}

Keywords : Aorta and great vessels, Cardiac Tumors, Aortic Surgery

\section{Key Clinical Message:}

- Difficult diagnosis due to rarity of the case

- TT or TE Echocardiography is sufficient to make a correct diagnosis

- Urgent surgical resection is mandatory due to the risk of embolism

\section{Introduction:}

Primary aortic wall tumors are very rare and consist mainly of malignant tumors ${ }^{1}$. Papillary fibroelastomas (PFE) are benign neoformations usually originating from the valvular endocardium, consisting of non-vascularized papillary tissue surrounded by an endothelium line ${ }^{2}$. The localization of PFE on the aortic wall is extremely rare $^{3},{ }^{4}$. The clinical presentation of aortic PFE is usually related to embolization with myocardial infarction and/or stroke ${ }^{4,5}$. We report a case of aortic wall PFE simulating unstable angina, diagnosed with trans-thoracic and trans-esophageal echocardiography (TTE/TEE) and surgically removed.

\section{Case Report}

A 73-year-old woman, hypertensive and diabetic, came to ER with typical angina symptoms. The patient had undergone PTCA with DES on the anterior descending and first diagonal coronary arteries, following acute myocardial infarction, 3 years before. Subsequently, one and a half years later a further coronary artery examination, performed after a chest pain episode with positive ECG, showed a good success of the previous PTCA's. No other coronary lesions were found and nothing was detected in the ascending aorta. At the current admission the ECG showed ST depression in II-III-aVF that spontaneously resolved and there was no significant HS troponin enzymatic release. At a routine TTE the presence of a mass in the upper part of the right coronary sinus just above the right coronary ostium was noted. A TEE was quickly performed confirming the presence of a mobile mass of about $1 \mathrm{x} 1.5 \mathrm{~cm}$ in diameter with irregular profile (Figure 1A). A CT scan was then performed confirming the location, aspect and size of the mass (Figure 1B). Although the patient remained asymptomatic, the mobility of the mass associated with the previous episode of chest pain made us put an indication to urgent surgery.

After a median sternotomy the extracorporeal circulation was started by cannulation of the ascending aorta and left atrium. After aortic clamping, longitudinal aortic root exposure was obtained. A transparent gelatinous mass was found, implanted by a small peduncle in the upper part of the right coronary sinus slightly laterally to the coronary ostium, which appeared partially covered by the mass itself (Figure 2A). The tumor was excised at the base, without the need to remove part of the aortic root (Figure 2B). The total CPB time was $32 \mathrm{~min}$ and the cross clamp $16 \mathrm{~min}$. The postoperative course was uncomplicated and the patient discharged home on the seventh POD. Before discharge a new coronary artery angiography showed an unchanged finding. The histopathological examination confirmed that the mass was a papillary fibroelastoma without thrombotic component (Figures 2C).

\section{Discussion}

PFE are second in frequency, after myxomas, among primary cardiac tumors, representing about $10 \%$. They present macroscopically with the appearance of a sea anemone with leafy arms, starting from a stalked

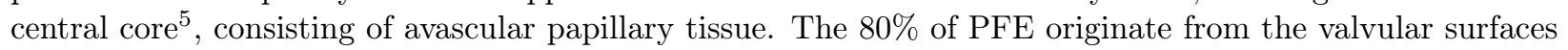
and the remaining $20 \%$ from other cardiac localizations; aortic localization is extremely rare and can occur 
in all aortic segments. To the best of our knowledge, only three cases of aortic wall PFE have been described in literature ${ }^{3,6,7}$.

In aortic wall PFE, although the majority of patients remain asymptomatic, clinical onset usually occurs with cerebral ischemic or coronary symptoms due to mass embolization or partial or total coronary occlusion ${ }^{3,4,6,7}$. The diagnosis is very difficult mainly due to their low frequency and consequently the lack of knowledge of the case; this is the unique case of aortic PFE reported in our center after about 20,000 cardiac interventions. In addiction presentation with angina symptoms in a patient with previous PTCA caused diagnostic confusion. Usually transthoracic echocardiography (TTE) is sufficient to allow a diagnosis with high sensitivity and specificity $(88.8 \% \text { and } 88.7 \% \text { respectively })^{8}$ where TEE has even greater sensitivity in smaller PFEs. The execution of a CT scan can be useful to define the diagnosis itself which, due to its extreme rarity, is hardly immediately evaluated. In fact, in our case the typical angina symptoms would probably have led us to perform a new coronary angiogram, with the associated embolic risk, given the difficulty to think of a PFE of the aorta. The presence of the mass, already evident in TTE, led us to the execution of TEE which allowed a diagnosis with high probability; the CT confirmed the size and position of the mass allowing us to place the clinical suspicion and the consequent surgical indication to the removal. Coronary angiography was not performed to avoid mobilization of the mass.

There are no current guidelines for the treatment of PFE. In patients with embolic events, surgery is strongly recommended $^{9}$. In asymptomatic patients the opinions are different and are based on mass mobility, position, concomitant surgery etc. We believe that, since the tumor is positioned in the aortic flow, surgery should always be indicated for the potential embolic risk, except in asymptomatic highrisk patients. Given the benign nature of the tumor a simple but accurate excision is sufficient. After surgical resection no recurrence of the tumor has ever been reported ${ }^{6}$.

\section{Conclusions}

PFEs of the aortic wall are exceptionally rare and can be found accidentally or show symptoms of cerebral or cardiac ischemia. The diagnosis is particularly difficult mainly due to the lack of experience of the operators. TTE/TEE is the method of choice for their diagnosis while CT scan or MRI can be helpful in the diagnostic doubt. Surgical resection, where possible, is mandatory due to the risk of embolism and is generally low risk surgery. Our case highlights how clinical suspicion should be placed even when the mass is in such atypical positions.

\section{Disclosure Statement}

No conflict of interest to declare

\section{Author contributions}

Concept/design PM,GM,GE

Drafting article: PM, PC, II, ADR, GC, DT

Critical revision of article: SI, GM, AC

Ethics approval and consent to participate : ethical approval was obtained from the director of A.O.U. San Giovanni di Dio e Ruggi D'Aragona Hospital. For case reports is not necessary a formal approval in this hospital

Consent to publish : the written consent for publication was released by the patient

Availability of data and materials : the dataset generated during the current study are not publicly available because of privacy regulation but are available from the corresponding author on reasonable request

Funding: the authors declare that not founding sources were utilized

\section{REFERENCES}


1. Das AK, Reddy KS, Suwanjindar P, Fulmer A, Siquiera C Jr, Floten S et al. A. Primary Tumors of the Aorta. Ann Thorac Surg 1996;62:1526-8

2. Burke A. Tumors of the heart and great vessels. Atlas of tumor pathology 1996;16:171-9

3. Yerebakan C, Liebold A, Steinhoff G, Skrabal CA. Papillary Fibroelastoma of the Aortic Wall With Partial Occlusion of the Right Coronary Ostium. Ann Thorac Surg 2009;87:1953-

4. Rolf T, Iglesias JF, Tozzia P, von Segessera LK. Acute myocardial infarction caused by coronary embolization of a papillary fibroelastoma of the thoracic ascending aorta. Interactive CardioVascular and Thoracic Surgery 11 (2010) 676-678

5. Klarich KW, Enriquez-Sarano M, Gura GM, Edwards WD, Tajik AJ, Seward JB. Papillary fibroelastoma: echocardiographic characteristics for diagnosis and pathologic correlation. J Am Coll Cardiol 1997;30:784-90

6. Anand S, Sydow N, Janardhanan R. Papillary fibroelastoma diagnosed through multimodality cardiac imaging: a rare tumour in an uncommon location with review of literature. BMJ Case Reports 2017(8);2017:bcr-2017-219327

7. Gonzalez-Santos JM, Arnaiz-Garcıa ME, Vargas-Fajardo MC, Arribas-Jimenez A. Aortic wall papillary fibroelastoma J Thorac Cardiovasc Surg 2013;146:e1-3

8. Sun JP, Asher CR, Yang XS, Cheng GG, Scalia GM, Massed AG et al. Clinical and echocardiographic characteristics of papillary fibroelastomas: a retrospective and prospective study in 162 patients. Circulation 2001;103:2687-93.

9. Gowda RM, Khan IA, Nair CK, Metha NJ, Vasadava BC Sacchi TJ. Cardiac papillary fibroelastoma: a comprehensive analysis of 725 cases. Am Heart J 2003;146:404-10.

\section{LEGENDS}

Figure 1. A: TEE showing a mobile, irregular mass of 1x1,5 cm diameters in right coronary sinus. B: CTA scan confirming the presence of the mass in ascending aorta

Figure 2. A: Surgical view of the mass. B: Macroscopic view of the resected mass. C: The histology shows individual fronds consisting of a core of hyalinized hypocellular stroma that is rich in elastic fibers and a lining of hyperplastic endocardial cells (magnification ratio:200:1 - 400:1. Haematoxylyn eosin staining)

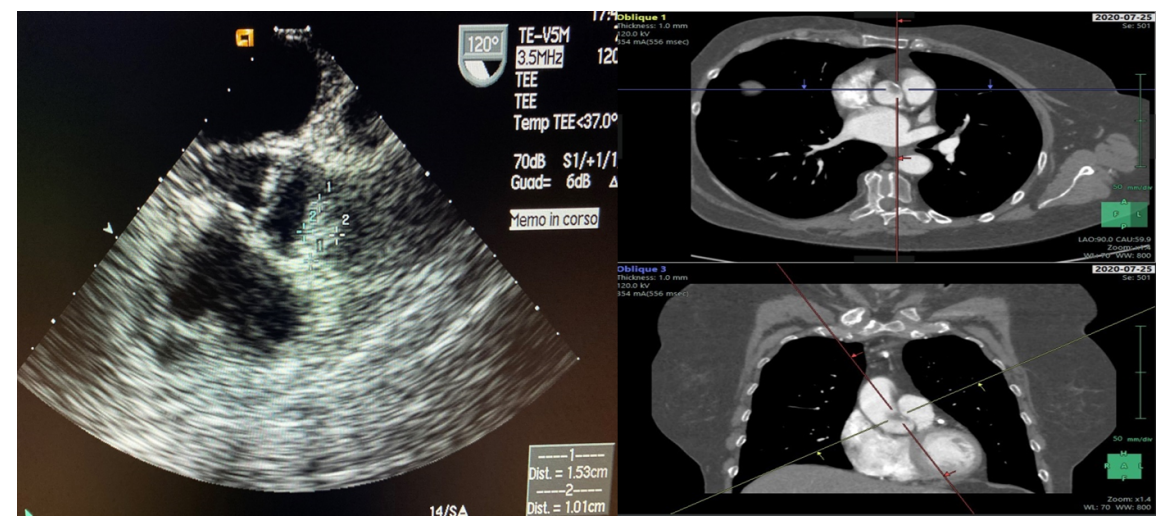




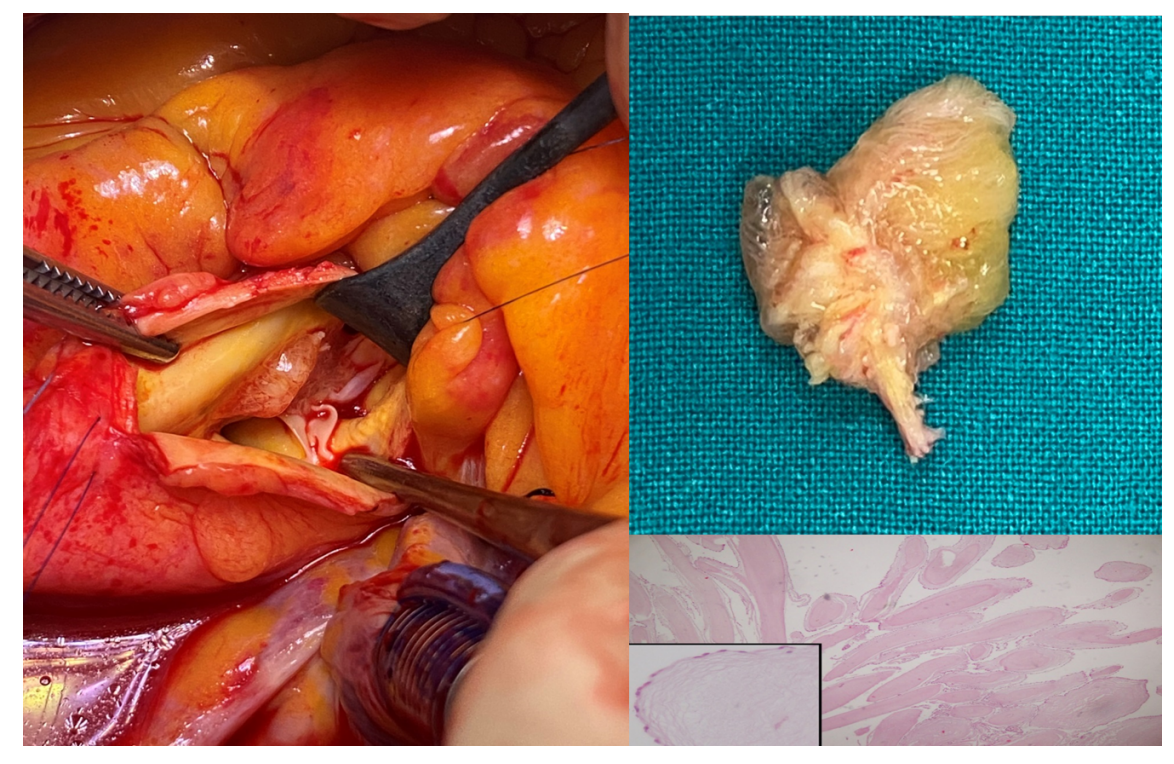

